\title{
JE PARLE DONC JE SUIS... CONFIGURATIONS IDENTITAIRES SUR FOND DE LANGUES
}

\author{
MARIA ZERVA \\ Université de Strasbourg, France
}

\begin{abstract}
Résumé. Les langues sont de puissants symboles d'identité et contribuent à tracer des frontières entre les individus et les groupes, mais l'inverse est également vrai : les individus ou les groupes circonscrivent et classent les langues dans le cadre de leur construction identitaire. De plus, définir sa propre maîtrise d'une langue, par exemple la minorer, est un moyen de construction identitaire et de négociation. Dans cet article, nous nous concentrons sur la construction discursive de l'identité des Grecs ayant le turc comme langue d'origine. Nous montrons comment les macro-choix (par exemple, l'abandon d'une langue) ou les micro-choix, comme les choix linguistiques dans une phrase, constituent l'agentivité des individus et des groupes dans leur dialogue avec les discours dominants.
\end{abstract}

Mots-clés : construction identitaire, analyse de discours, sociolinguistique

\section{INTRODUCTION}

Si les langues, importants symboles identitaires (Blanchet, 2000), contribuent à tracer des frontières entre les individus et les groupes (Tabouret-Keller, 1997), l'inverse n'est pas moins vrai : les individus ou les groupes tracent des frontières entre langues, les circonscrivent et les classifient en fonction de leurs affiliations identitaires. Il s'agit là d'un moyen de construction identitaire et de construction de l'altérité, la deuxième étant fondamentale pour la première. De même, situer sa propre maitrise d'une langue, la revendiquer ou la sous-estimer, se révèle également être un moyen à disposition du sujet pour construire et négocier son identité et son rapport à l'Autre et au monde. Dans cet article, nous nous pencherons sur des extraits de discours recueillis lors d'entretiens semi-directifs auprès de Grecs ayant comme langue d'origine la langue de l'Autre national, à savoir le turc. Une analyse de discours fine nous permettra de déceler les moyens discursifs de construction de représentations identitaires dans et par le discours (voir, entre autres, Matthey, 2000 ; Boyer, 2003).

Notre terrain de recherche est constitué par deux communautés (deux villages) en Grèce, l'une au nord-ouest du pays, près de la ville de Yannina (Bafra) 
et l'autre au nord-est, près de la ville de Serrès (Nea Bafra). Ces villages ont été fondés entre 1924 et 1925 par des réfugiés d'Asie Mineure venus en Grèce à la suite du Traité de Lausanne (1923), prévoyant un échange de populations obligatoire entre la Grèce et la Turquie sur la base de la religion : tous les chrétiens orthodoxes de la Turquie devaient gagner la Grèce et tous les musulmans de Grèce la Turquie, à quelques exceptions près. L'échange de populations prévu aux marges de ce Traité a été massif et a profondément marqué le destin de la Grèce et les esprits de ses habitants ; il a concerné environ 1,2 million de personnes, arrivées dans un pays qui comptait à peine 4,5 millions (pour une introduction, voir, entre autres, Hirschon, 2003).

Parmi ce 1,2 million de réfugiés, il y avait 100.000 personnes turcophones ignorant le grec. Pour eux, l'expérience du déracinement, de toute façon douloureuse pour l'ensemble des populations, devait être encore plus difficile, en raison de la turcophonie, la langue turque étant incompatible avec le modèle identitaire grec, selon lequel un Grec doit être grécophone et chrétien orthodoxe (Zerva, 2010). Les Grecs turcophones orthodoxes ont donc développé des stratégies de compensation de ce 'défaut' pour faciliter leur intégration revendiquée dans la nation grecque. Au centre de ces stratégies se trouvait l'assimilation linguistique, à savoir l'abandon de la langue turque, mais aussi la sur-accentuation de l'appartenance religieuse et un discours martyrologique (Zerva, 2011). Dans le cadre de ce dernier s'intègre l' histoire-mythe' (Marantzidis, 2001), un récit, non prouvé historiquement, qui circule auprès de ces populations et explique la turcophonie comme un choix contraint, fait par les ancêtres à l'époque de l'empire ottoman, lorsque les Turcs les ont obligés à choisir entre leur langue (naturellement, le grec) et leur religion : ils ont choisi la religion, élément national primordial. Ainsi, la turcophonie se trouve justifiée, tandis que l'adoption du grec est envisagée comme un retour à l'état naturel'.

\section{CADRE THEORIQUE ET METHODOLOGIQUE}

L’enquête de terrain dans les deux communautés a été effectuée au moyen d'entretiens semi-directifs avec un guide d'entretien servant de trame à la conversation. Nous avons été en février 2005 à Bafra et en avril 2007 à Nea Bafra, où nous avons fait 36 entretiens de $922,5 \mathrm{~min}$. au total (Zerva, 2011). Nos interlocuteurs étaient des personnes d'origine réfugiée de $2^{\text {ème }}, 3^{\text {ème }}$ et $4^{\text {ème }}$ génération. L'objectif de ce travail de recherche a été de mettre en évidence la situation sociolinguistique de Bafra et de Nea Bafra, de suivre l'évolution de l'assimilation linguistique dans le temps et de déceler le processus de construction identitaire dans et par le discours. Etant consciente que notre corpus comprend des entretiens, à savoir du discours sur (épilinguistique), des déclarations pouvant être en décalage par rapport aux pratiques effectives, nous avons choisi de travailler avec la notion des représentations sociales (dorénavant RS), avec laquelle les sociolinguistes francophones ont l'habitude de travailler.

Définie comme 'une forme de connaissance, socialement élaborée et partagée, ayant une visée pratique et concourant à la construction d'une réalité 
commune à un ensemble social' (Jodelet, 1989 : 36), la notion de RS présente l'avantage d'insister sur le fait que l'humain ne détient pas la réalité objective mais la construit, en se la représentant. Ce processus cognitif est accessible par sa mise en discours, qui nous permet de suivre la dynamique de la construction des RS (Moscovici, 2001: 29 ; Matthey, 2000: 23 ; Py, 2004 : 6-7). Pour le repérage des RS, nous avons combiné l'analyse de contenu thématique et une analyse discursive, inspirée des outils fournis par la linguistique de l'énonciation (Kerbrat-Orecchioni, 2002/1980), l'analyse du discours (cf. Mainguenau, 1991) et l'analyse critique du discours (cf. Wodak, de Cillia, Reisigl et Liebhart, 1999).

La construction des RS est inséparable d'une mémoire intertextuelle/ interdiscursive (Adam, 1999 : 105) et ne dépend pas uniquement des discours présents, mais aussi antérieurs, du dialogue avec d'autres, qu'ils soient réels ou imaginaires, et de la polyphonie de toutes ces voix (Bakhtine et Volochinov, 1977). En même temps, les discours 'localisés', produits hic et nunc, rentrent en dialogue aussi avec des Discours dominants, en s'y opposant ou en y adhérant. Par Discours dominants, nous entendons des ensembles de discours culturellement partagés qui circulent dans une société, alimentent et, en même temps, contraignent la production discursive locale des sujets (le discours avec minuscule) (cf. Kiesling, 2006 : 261-265 ; De Fina, Schiffrin et Bamberg, 2006 : 7). Ces Discours sont étroitement liés aux idéologies dominantes (Van Dijk, 2008) et sont liés à la mémoire interdiscursive mentionnée.

Dans et par le discours sont aussi construites les identités, considérées comme une construction sociale et discursive constante, un processus dynamique, et non pas une caractéristique innée aux individus et aux groupes. Les identités se construisent dans une constante négociation entre l'individu et les structures sociales qui l'englobent, ainsi que les autres individus et groupes (Benwell et Stokoe, 2006 : 17-47 ; Wodak et al., 1999 : 1-48 ; Mendoza-Denton, 2002 : 475). Selon le contexte, l'individu se positionne et est acteur, il a une capacité d'action (agentivité ; agency), malgré les contraintes du contexte (Holland, Lachicotte, Skinner et Cain, 1998 ; Wodak et al., 1999). C'est dans ce sens que nous examinerons les choix des populations en question, pour déceler comment leurs choix sur le plan macro, comme la loyauté à une variété linguistique, mais aussi sur le plan micro, en tant que mise en discours, font partie de leur construction identitaire.

\section{CONSTRUIRE SON IDENTITE AVEC ET MALGRÉ LE TURC}

Nous avons, dans l'Introduction, mentionné les principales stratégies mises en place pour intégrer l'élément 'problématique' qu'est le turc dans la construction identitaire des descendants des turcophones orthodoxes en tant que Grecs à part entière, à savoir l'assimilation linguistique, la sur-accentuation de la religion et le discours martyrologique. Ajoutons à ceux-là la minoration du turc (Blanchet, 2000) et les efforts constants de prise de distance avec cette langue. Le turc est ainsi présenté comme une contrainte, son acquisition ayant été obligatoire et ne 
relevant pas d'un choix. En outre, la maîtrise du turc donne lieu à une justification, comme l'histoire-mythe, à des expressions de gêne, comme des rires, ou à des commentaires, comme par exemple, 'c'est resté gravé dans ma tête, je ne peux pas l'oublier'. De plus, la grande majorité des personnes parlant turc minorent leurs propres compétences et disent soit ne pas bien le parler soit l'avoir oublié. Enfin, on minore le turc du village, qui est caractérisé comme une langue quotidienne, éloignée du turc 'pur' de Turquie. Si l'on se pose la question de savoir s'il s'agit là de marques d'insécurité linguistique, nous répondrions par la négative : il s'agirait plutôt d'une stratégie identitaire de prise de distance avec l'identité turque et d'une construction identitaire positive, majorée, en adéquation avec les Discours dominants grecs.

Nous allons maintenant nous concentrer sur un long extrait monologique tiré d'un de nos entretiens avec un homme de 81 ans de Nea Bafra (NBm81), membre des élites locales, qui raconte l'histoire-mythe', ce récit qui résout la contradiction entre turcophonie et identité grecque et justifie, en même temps, l'authenticité de la grécité des Grecs turcophones. Pour des raisons de place, nous nous permettons de le reproduire uniquement dans sa traduction française.

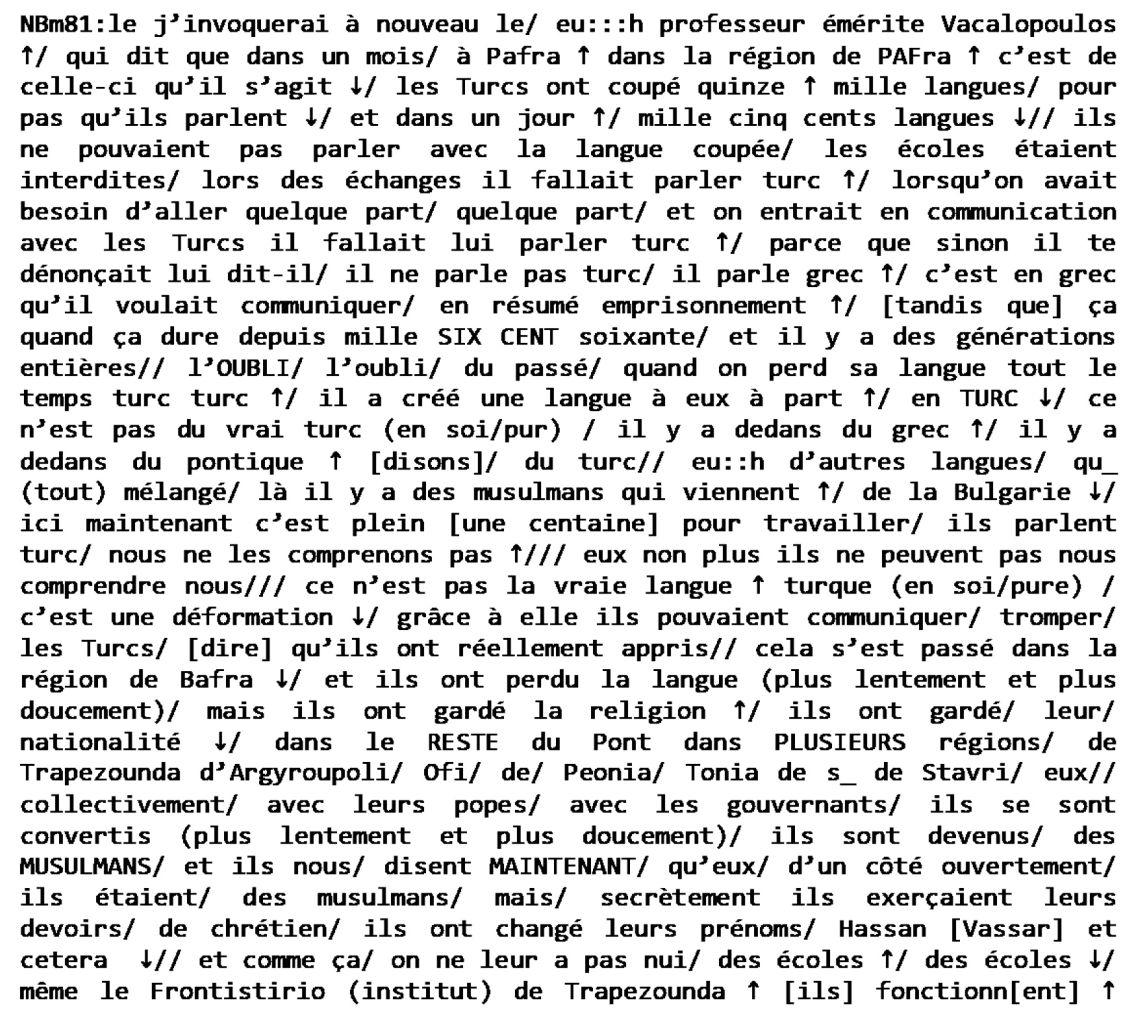


Malgré la multitude d'éléments que nous pourrions commenter à propos de cet extrait, comme le modèle temporel ou l'usage des temps verbaux, nous nous concentrerons sur les lignes 13 à 16 et 20-21, dans lesquelles notre interlocuteur développe 'l'astuce' trouvée par les ancêtres pour déjouer l'ennemi qui les aurait obligés à renoncer au grec et à parler turc : ils ont donc 'fait semblant' d'obéir, mais au lieu de vraiment parler turc, ils ont créé une langue mixte, distincte du turc, avec des éléments grecs, grecs pontiques (dialecte du grec) et turcs. Voyons maintenant les détails d'une analyse discursive fine.

Dans la ligne 13, NBm81 dit : 'il/on a créé une langue à eux à part (distincte) $\uparrow /$ en TURC $\downarrow /$ ' (dimiourgise mia diki tous ksehoristi glossa $\uparrow /$ sta TOURKIKA $\downarrow /$; notons que les parenthèses dans le texte français marquent une traduction alternative ou une explication). Il insiste bien sur le caractère distinct de cette langue à l'aide de plusieurs moyens linguistiques : a) le sémantisme du verbe créer, soulignant l'invention, l'innovation; b) l'adjectif distinct (en grec ksehoristi, qui peut aussi être traduit comme à part) qui n'est pas axiologique (même s'il est souvent connoté positivement), mais comme il contribue au résultat désiré (la dissociation du turc), il se charge, dans ce contexte, d'une évaluation axiologique positive ; $\mathrm{c}$ le déterminant indéfini une qui renvoie à une opération d'extraction selon la théorie culiolienne et d) le 'pronom possessif emphatique et déterminant' (à eux, placé avant l'adjectif en grec) (Holton, Mackridge et Philippaki-Warburton, $1998: 100$ et 323 ; cf. aussi Clairis et Babiniotis, 2005 : 295) qui renvoie, lui, à une opération de fléchage. Le caractère distinct de cette langue hybride est alors de nouveau souligné par ces deux opérations: avec l'extraction, elle 'acquiert un statut existentiel - réel ou imaginaire -' (TsamadouJacoberger, 1998 : 100), qui la distingue de la classe 'langue turque'. Avec le fléchage, elle est expressément qualifiée comme étant différente, distincte. e) Cette nouvelle langue n'est pas explicitement nommée ; on ne dit pas qu'on a créé une langue distincte qui est turque, mais qu'on a créé une langue distincte en turc. f) Enfin, l'intonation et la gestion des pauses (marquées par le symbole /) semble contribuer aussi à la distinction entre turc et nouvelle langue hybride.

Par la suite, NBm81 explique que 'ce n'est pas $d u$ vrai/pur turc [ce n'est pas du turc en soi] / il y a dedans du grec $\uparrow /$ il y $a$ dedans du pontique $\uparrow$ [disons]/ du turc// eu::h d'autres langues/ qu_(tout) mélangé/'. La création d'une langue hybride, distincte du turc est ici explicitement posée, tandis que le rôle du grec dans le mélange est mis en avant (avec le grec et le grec pontique au début et avec une intonation ascendante) et celui du turc minoré, arrivé à la fin et entouré de marqueurs d'hésitation (disons, euh). Or, si le produit est un mélange (en grec berdemena), donc minoré, le processus est bien majoré, comme cela devient clair un peu plus loin. Après un récit dans le récit (lignes 16-19), dont le rôle est d'appuyer les propos (nous ne nous comprenons pas avec les travailleurs saisonniers musulmans turcophones qui viennent ici), le locuteur continue : 'ce n'est pas la vraie/pure langue $\uparrow$ turque (en soi)/ c'est une déformation (falcification) $\downarrow /$ grâce à elle (cette langue hybride) ils pouvaient communiquer/ tromper/ les Turcs/ (dire) qu'ils ont réellement appris// [...]'. Ici le locuteur 
a recours à la répétition, puisqu'il reprend presque la même phrase que dans la ligne 14. Ainsi, il veut non seulement renforcer la force assertive de son énoncé, mais aussi d'impliquer son interlocuteur dans sa construction, dans le but de le persuader (Tsakona 2009: 83-85 et 93). En ce qui concerne les choix linguistiques dans la première partie ('ce n'est pas la vraie/pure langue $\uparrow$ turque (en soi)/ c'est une déformation (falcification) $\downarrow$ ', den ine i glossa $i$ katheaftou $i$ tourkiki/ ine mía parapiisi $\downarrow /$ ), il est intéressant de constater le choix du déterminant défini avant chaque nominal (nom, adjectif et adverbe nominalisé). La structure de la première phrase se présente ainsi :

Litt. Ce n'est pas la langue la pure (en soi) la turque... (adj.).

Nég. $+\mathrm{V}+$ Dét. déf. + N1 (nom) + Dét. déf. + N2 (adv.) + Dét. déf. + N3

L'introduction de chaque nominal par un déterminant défini marque, en termes culioliens, une qualification différentielle ou opération de fléchage qui produisent un effet de contraste (Tsamadou-Jacoberger, 1998 : 148-151). Ainsi, on produit une gradation dans la 'turcité' de la langue ; la langue hybride se trouve en dehors du domaine de la langue turque pure, mais à l'intérieur tout de même d'une notion de la langue turque autre que pure ; ainsi on ne s'éloigne pas de l'évidence.

\section{CONCLUSIONS}

Nous avons vu comment les Grecs orthodoxes prennent des distances avec le turc sur le plan macro, mais aussi micro. Dans les extraits examinés, la turcophonie est présentée comme un acte de résistance qui contribue finalement à la majoration du groupe. Ainsi, l'individu adhère aux Discours dominants nationaux, à l'idéologie nationale, mais ne reste pas passif. Il les négocie et déploie son agentivité (agency) (cf. Holland et al., 1998) pour construire son identité dans les meilleures conditions possibles (être des Grecs à part entière) et tirer le maximum des bénéfices. Cette agentivité lui permet de trouver l'équilibre entre adhésion aux Discours dominants et construction d'une identité majorée, positive, sans pour autant renier complètement l'histoire du groupe et s'auto-déprécier.

\section{BIBLIOGRAPHIE}

Adam,J.-M. (1999) Images de soi et schématisation de l’orateur : Pétain et De Gaulle en juin 1940. In R. Amossy (dir.) Images de soi dans le discours. La construction de l'ethos (pp. 101-126). Lausanne-Paris: Delachaux et Niestlé.

Bakhtine, M. (Volochinov, V. N.) (1977) Le marxisme et la philosophie du langage. Paris: Les Editions de Minuit.

Benwell, B. et Stokoe, E. (2006) Discourse and Identity. Edinburgh: Edinburgh University Press. Blanchet, P. (2000) La linguistique de terrain. Méthode et théorie. Une approche ethnosociolinguistique. Rennes: Presses Universitaires de Rennes.

Boyer, H. (2003) De l'autre côté du discours. Recherches sur les représentations communautaires. Paris: L'Harmattan. 
Clairis, C. et Babiniotis, Y. (2005) Grammaire du grec moderne. Structurelle - fonctionnelle communicationnelle (en grec). Athènes: Ellinika Grammata.

De Fina, A., Schiffrin, D. et Bamberg, M. (éds.), (2006) Discourse and Identity. Cambridge: Cambridge University Press.

Hirschon, R. (éd.), (2003) Crossing the Aegean. An appraisal of the compulsory population exchange between Greece and Turkey. New York, Oxford: Berghahn Books.

Holland, D., Lachicotte Jr., W., Skinner, D. et Cain, C. (1998) Identity and Agency in Cultural Worlds. Cambridge, Massachusetts / London, England: Harvard University Press.

Holton, D., Mackridge, P. et Philippaki-Warburton, I. (1998) Grammaire de la langue grecque (en grec). Athènes: Patakis. (1ère édition en anglais en 1997).

Jodelet, D. (1989) Représentations sociales : un domaine en expansion. In D. Jodelet (dir.) Les représentations sociales (pp. 31-61). Paris: Presses Universitaires de France.

Kerbrat-Orecchioni, C. ([1980] 2002) L'énonciation; de la subjectivité dans le langage. Paris: Armand Colin.

Kiesling, S. F. (2006) Hegemonic identity-making in narrative. In A. de Fina, D. Schiffrin et M. Bamberg (éds.) Discourse and Identity (pp. 261-287). Cambridge: Cambridge University Press.

Maingueneau, D. (1991) L'analyse du discours. Introduction aux lectures d'archive. Paris: Hachette.

Marantzidis, N. (2001) Yassassin Millet. Vive la nation. Réfugiés, Occupation et Guerre Civile: Identité ethnique et conduite politique des turcophones grecs-orthodoxes du Pont Occidental (en grec). Héraklion: Presses Universitaires de Crète.

Matthey, M. (2000) Aspects théoriques et méthodologiques de la recherche sur le traitement discursif des représentations sociales. TRANEL, 32: 21-37.

Mendoza-Denton, N. (2002) Language and identity. In J. K. Chambers, P. Trudgill et N. ShillingEstes (éds.) The Handbook of Language Variation and Change (pp. 475-499). Oxford (UK) / Malden (USA) / Victoria (Australia): Blackwell Publishing.

Moscovici, S. (2001) Why a Theory of Social Representations? In K. Deaux et G. Philogène (éds.) Representations of the Social (pp. 8-35). Oxford, Massachussets: Blackwell Publishers.

Py, B. (2004) Pour une approche linguistique des représentations sociales. In J.-C. Beacco (dir.) Représentations métalinguistiques ordinaires et discours. Langages, 154: 6-19.

Tabouret-Keller, A. (1997) Language and Identity. In F. Coulmas (éd.) The Handbook of Sociolinguistics (pp. 315-326). Oxford, Massachusetts: Blackwell Publishers.

Tsakona, V. (2009) Linguistic Creativity, Secondary Orality, and Political Discourse: The Modern Greek Myth of the 'Eloquent Orator'. Journal of Modern Greek Studies, 27 (1): 81-106.

Tsamadou-Jacoberger, I. (1998). Le nom en grec moderne. Marqueurs et opérations de détermination. Paris, Montréal: L'Harmattan.

Van Dijk, T. (1998) Ideology. A multidisciplinary approach. SAGE Publications.

Wodak, R., de Cillia, R., Reisigl, M. et Liebhart, K. (1999) The discursive construction of national identity. Edinburgh: Edinburgh University Press.

Zerva, M. (2011) Les Grecs turcophones orthodoxes : une étude sociolinguistique. Thèse de doctorat. Université de Strasbourg. Vol. I et Vol. II.

Zerva, M. (2010) Le citoyen grec par excellence. Conception normative du citoyen grec par l'Etat-nation grec et cas de marginalité. In N. Abi-Rached (dir.) Normes et marginalités à l'épreuve (pp. 49-61). Strasbourg : Presses Universitaires de Strasbourg. 


\title{
I SPEAK THEREFORE I AM... IDENTITY CONFIGURATIONS BASED ON LANGUAGES
}

\begin{abstract}
Languages are powerful identity symbols and contribute to the drawing of borders between individuals and groups, but the opposite is also true: individuals or groups circumscribe and classify languages as part of their identity construction. Moreover, to define his/her own command of a language, for example, underestimate it, is also a means of identity construction and negotiation. In this paper, we focus on the discursive construction of identity of Greeks having Turkish as their heritage language. We show how macro choices (e.g. language shift) or micro ones, like the linguistic choices in a phrase, constitute individuals' and groups' agency in their dialogue with dominant discourses.
\end{abstract}

Key words: identity construction, discourse analysis, sociolinguistics

Maria Zerva (Maître de Conférences en Etudes néo-helléniques) travaille à l'Université de Strasbourg. Ses recherches et publications se concentrent sur le multi- et le plurilinguisme, les idéologies dominantes, le nationalisme, les identités, les représentations sociales, l’analyse de discours. Courriel : mzerva@ unistra.fr 